\title{
TEICHMANN prueba confirmativa para identificación de sangre en manchas
}

\author{
TEICHMANN confirmative test for blood identification in stains
}

\author{
N. Castillo-Rodríguez iD; S. Martinez-Garcia iD
}

\begin{abstract}
The analysis of blood identification in dry spots is carried out using the Teichmann crystallographic method as a basis to the colorimetric orientation tests in the expert work of the Forensic Biology laboratories of the National Institute of Legal Medicine and Forensic Sciences To determine by Teichmann's crystallographic method its sensitivity, specificity, positive predictive value, negative predictive value, limit of detection, concordance index and Kappa index according to the different conditions of temperature, support and medium in which they are located. 100 samples of dry spots are taken to be analyzed double-blind, in different substrates using the Teichmann confirmatory method for blood identification, following the adequate biosecurity measures by the Forensic Biology Laboratory. The statistical quality values obtained in sensitivity were $96,22 \%, 100 \%$ Specificity, $100 \%$ Positive Predictive Value, 96,87\% Predictive Negative Value, 1/100 Detection Limit, $\mathbf{1 0 0 \%}$ Concordance Index and a Kappa Index. of 1 . Through the data obtained, the effectiveness of Teichmann's crystallographic method as a confirmatory test in support of presumptive methods of orientation in the identification of blood in spots is made manifest.
\end{abstract}

Index Terms - Confirmatory, Crystallization, Specificity, Forensic, Hemochromogen, Blood.

Resumen-Se realiza el análisis de identificación de sangre en manchas secas utilizando el método cristalográfico de Teichmann como fundamento a las pruebas colorimétricas de orientación en la labor pericial de los laboratorios de Biología Forenses del Instituto Nacional de Medicina Legal y Ciencias Forenses. Determinar por el método cristalográfico de Teichmann su sensibilidad, especificidad, valor predictivo positivo, valor predictivo negativo, límite de detección, índice de concordancia e índice de Kappa según las distintas condiciones de temperatura, soporte y medio en el cual se encuentren. Se toman 100 muestras de manchas secas para ser analizadas a doble ciego, en diferentes sustratos utilizando el método confirmativo de Teichmann para identificación de sangre, siguiendo las medidas de bioseguridad adecuadas por el laboratorio de biología Forense. Los valores estadísticos de calidad arrojados en sensibilidad fueron de $\mathbf{9 6 , 2 2 \%}$, Especificidad 100\%, Valor Predictivo Positivo $100 \%$, Valor Predictivo Negativo 96,87\%, Limite de Detección de 1/100, Índice de Concordancia del $100 \%$ y un índice de Kappa de 1. Por

Este manuscrito fue enviado el 08 de julio de 2019 y aceptado el 13 de marzo de 2020

N. Castillo-Rodríguez, Docente del Departamento de Física de la Universidad Tecnológica de Pereira (nancycastillo@utp.edu.co).

S. Martinez-Garcia, docente en la Facultad de Ingenierías en el Programa de Ingeniería Física de la Universidad Tecnológica de Pereira. (smartinez@utp.edu.co) medio de los datos obtenidos se pone en manifiesto la efectividad del método cristalográfico de Teichmann

Palabras claves - Confirmativa, Cristalización, Especificidad, Forense, Sangre, Sensibilidad.

Resumo-Realiza-se a análise de identificação de sangue em manchas secas utilizando o método cristalográfico de Teichmann como base aos testes colorimétricos de orientação no trabalho pericial dos laboratórios de Biología Forense do Instituto Nacional de Medicina Legal e Ciências Forenses. Estabelecer através do método de cristalografía de Teichmann a sua sensibilidade, especificidade, valor preditivo positivo, valor preditivo negativo, límite de detecção, índice de concordância e índice de Kappa dependendo das diferentes condições de temperatura, suporte e meio no qual se encontrem. Levam- se 100 amostras de manchas secas para serem analisadas com dupla ocultação, em diferentes substratos utilizando o método confermativo de Teichmann para identificação de sangue, seguindo as medidas de biossegurança adequadas pelo laboratório de biologia Forense. Os valores estatísticos de qualidade jogados em sensibilidade foram de $96,22 \%$, especificidade $100 \%$, valor preditivo positivo $100 \%$, valor preditivo negativo $96,87 \%$, límite de deteção de $1 / 100$, índice de concordância do $100 \%$ e um índice de de Kappa de 1. Através dos dados obtidos se põe em manifesto eficácia do método cristalográfico de Teichmann. Palavras-chave- Confirmativo, Cristalização,
Especificidade, Forense, Sangue, Sensibilidade.

\section{INTRODUCIÓN}

$U_{1}^{\infty}$ NO de los fluidos que con mayor frecuencia llega a los laboratorios de Biología forense de los Institutos de medicina legal y ciencias forenses de nuestro país, es la sangre, la cual reviste gran importancia en el esclarecimiento de numerosas investigaciones delictivas y judiciales [1] [2].

Las manchas de sangre presentan aspectos muy diferentes, según sean recientes o antiguas, si la mancha es fresca su color es rojo vivo, pero pierde muy rápidamente esta característica y se oscurece progresivamente, llegando con el tiempo a presentar una coloración marrón negruzca cuando es un poco vieja. Estas diferencias de coloración corresponden a las modificaciones que sufre la hemoglobina encontrada en los glóbulos rojos de la sangre, al entrar en contacto con el aire durante su transformación lenta en hematina [3]. 
Los métodos más utilizados en el estudio de las manchas de sangre son las pruebas colorimétricas de orientación presuntiva, siendo estas de gran sensibilidad, pero pueden llegar a ser poco específicas, lo que con lleva a complementar los resultados con técnicas cristalográficas cuya especificidad complementan la sensibilidad de las pruebas de orientación [4].

Otto Funke (1851) Fisiólogo Alemán publicó una serie de artículos en los que describía el crecimiento de los cristales de hemoglobina mediante la dilución sucesiva de glóbulos rojos con un disolvente (agua pura, alcohol o éter), seguida de la evaporación lenta del disolvente de la solución [5].

Kolliker (1853) encontró cristales de hemocromógeno en sangre de perro, pez y pitón siendo solubles en álcali, en ácido acético y nítrico siendo idénticos a los propuestos por Funke [6].

Van Deen (1861) diseñó un método que consistía en definir la existencia de peroxidasas en la muestra a estudiar, promoviendo la oxidación de la tintura de guayaco por acción del oxígeno [7].

Teichmann (1853) identificó la aparición de Cristales de forma rómbica cuando la sangre fuese tratada con Ácido acético glacial y cloruro de sodio como prueba de la identidad de sangre.

Sutherland (1907) considero una prueba positiva de los cristales de Teichmann, como una prueba segura de la presencia de sangre en la tinción sospecha de estudio u análisis [5].

Masaeo Takayama, 1912 realizó la identificación de sangre en manchas secas utilizando piridina, glucosa e hidróxido de sodio, la combinación de estos reactivos dio como resultado la formación de cristales de hemocromógeno [4].

El método cristalográfico de Teichmann se basa en la descomposición de la hemoglobina por acción del calor, dando lugar a la formación de la hematina, la cual, en presencia de una solución de ácido acético glacial, se combina con el cloro de la sal para formar cloruro de hematina, quien posteriormente se cristaliza por enfriamiento [8].

El fundamento químico de la prueba da origen a los cristales a partir de una hidrolisis acida, seguida de la oxidación de $\mathrm{Fe}+2$ a $\mathrm{Fe}+3$ de la hemina, formando cristales romboédricos de hemina o clorato de ferriprotoporfirina [2].

Hematina + Ácido Acético $+\mathrm{Cl} \rightarrow$ Clorato $\mathrm{Fe}$ Ferriprotoporfirinica (cristales de Hemina).

En la fig.1 se evidencia la estructura molecular del Clorato Ferriprotoporfirinica.

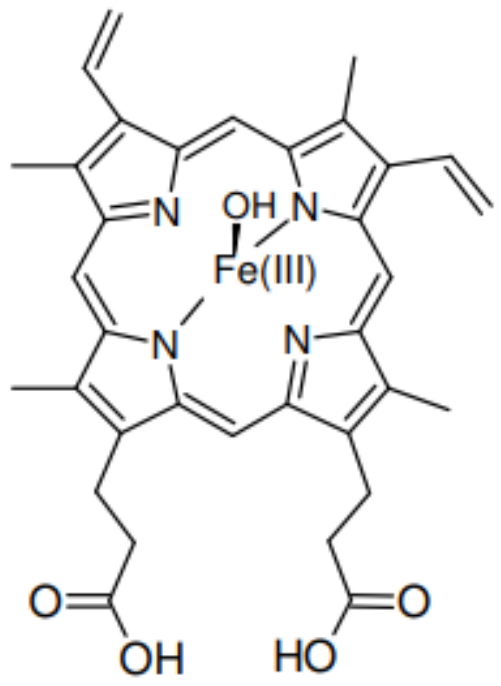

Fig.1 Estructura molecular del Clorato Ferriprotoporfirinica.

Los colores obtenidos en la reacción de confirmación de sangre en manchas en este método, pueden ser leves o intensos dependiendo de las condiciones de la prueba, la cantidad de la sustancia presente y la presencia de agentes reaccionantes [9].

El resultado de esta prueba se debe hacer por comparación de la sustancia desconocida con una sustancia de referencia o control bajo las mismas condiciones de temperatura, humedad y medio ambiente [10].

Los reactivos empleados en la preparación del Reactivo de Teichmann se realizaron siguiendo los procesos de bioseguridad establecidos por el Laboratorio de Biología del instituto Nacional de Medicina Legal y Ciencias Forenses [11].

Numerosos trabajos se han realizado en la investigación forenses de manchas de sangre [12] [13] [14] [15], las cuales con llevan a un continuo mejoramiento en pruebas de certeza para el aporte en los dictámenes del perito forense.

\section{MATERIALES Y METODOS}

\section{A. Materiales}

Algodón esterilizado, telas de varios colores, papel filtro, gradilla para tubos, pinzas metálicas de punta delgada, mechero de alcohol, laminas portaobjetos, laminas cubreobjetos, cajas de Petri (materiales para la preparación de las muestras); vasos de precipitación $100 \mathrm{ml}$, probeta de $10 \mathrm{ml}$, tubos de eppendorf de $1,5 \mathrm{ml}$, caja pesa sustancias, puntas desechables para pipetas automáticas, espátula delgada, frascos para almacenamiento de reactivos color ámbar, pipetas automáticas, tubos de ensayo, guantes, tapabocas, bolsas de papel pequeñas, bolsas plásticas y probeta graduada (materiales en la preparación de reactivos).

\section{B. Equipos Utilizados}

Cámara de extracción de gases, nevera $\left(5^{\circ} \mathrm{C}+/-3^{\circ} \mathrm{C}\right)$, 
destilador de agua, cronometro, balanza analítica, microscopio, horno con recirculación de aire, pipetas semiautomáticas con embolo. (Rango de 10ul, 200ul, 1000ul), termohigrómetro, vortex.

\section{Reactivos utilizados}

Hidróxido de sodio de pureza 99,9\%, Ácido acético glaciar de pureza $100 \%$, Agua destilada.

\section{Método}

En el análisis se utilizaron manchas de sangre secas en distintos sustratos, el método de estudio es una prueba confirmativa cristalográfica usando las medidas de bioseguridad adecuadas por el laboratorio de Biología forense.

\section{E. Población de estudio}

Se Tomaron 100 muestras entre manchas de sangre, puras y diluidas, extractos de productos vegetales, productos químicos y fluidos biológicos de origen humano, para su estudio con ordenamiento cruzado (muestras ya elaboradas, donde un analista asigna un código numérico a cada muestra y lo consigna en un listado, consecutivamente las muestras pasan a un segundo analista que las codifica por segunda vez y al finalizar se comparan los resultados obtenidos).

\section{F. Muestras}

En la Tabla I se observa la población de las muestras para su

TABLA I POBLACIÓN DE LAS MUESTRAS

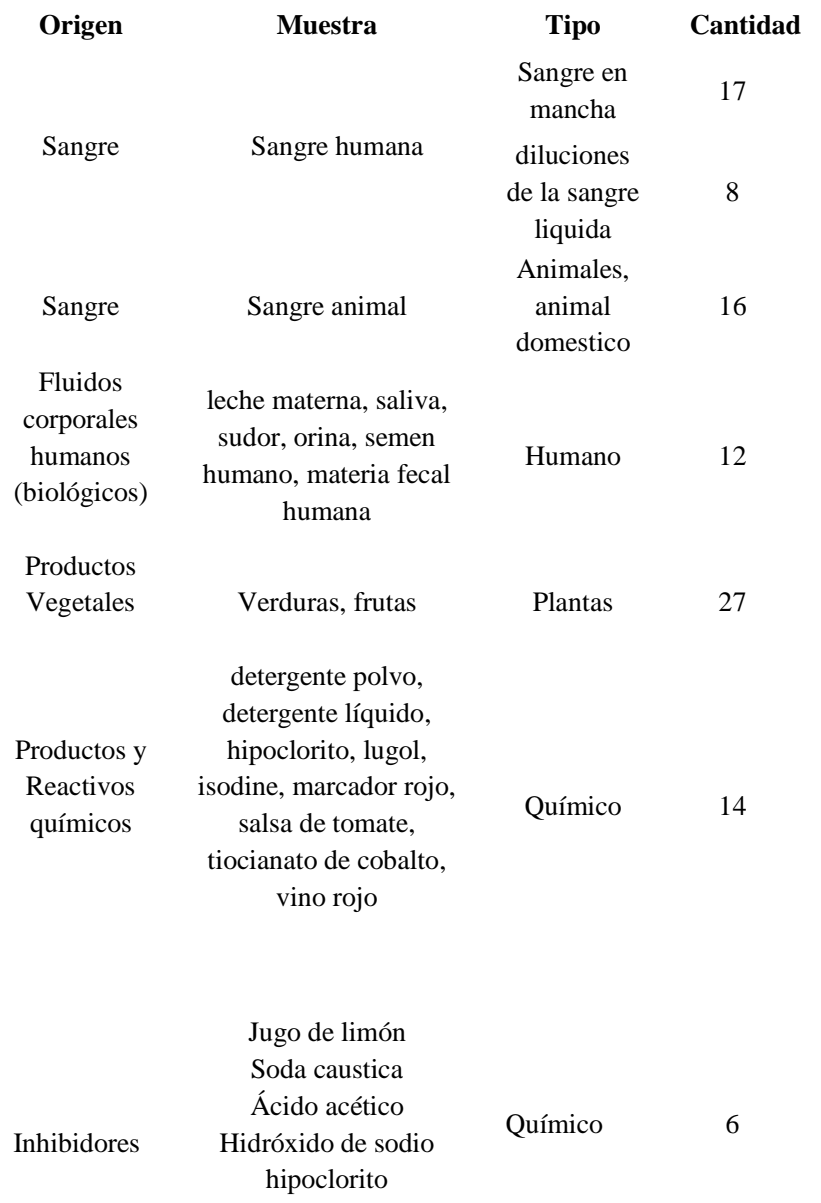

estudio.

\section{G. Criterios de selección de muestras}

Como control positivo se utilizó sangre humana y como control negativo agua salina de concentración $0,85 \%$, se obtuvieron muestras de sangre de animal doméstico y animales salvaje adoptando las medidas de salubridad pertinentes. Se seleccionaron fluidos corporales de origen humano con el propósito de poder identificar algún tipo de reacción con el método analítico en estudio y de esta manera llegar así a identificar las posibles interferencias. Los productos vegetales, sus derivados y los productos químicos fueron utilizados por su coloración similar a la de la sangre.

\section{H. Sustratos y elaboración de sustratos}

Se utilizaron sustratos como: tela garza por su característica absorbente y la no presencia de porosidad alguna, papel filtro, dacron, seda, nylon, jean, lino y franela, todos ellos de diferente color. La preparación de sustratos o mancha seca se llevó acabo; seccionando fragmentos de $0,5 \mathrm{~cm}$ x $0,5 \mathrm{~cm}$ se le adicionaron cuarenta microlitros 40ul del fluido a indagar, se pasó a secar en cabina de flujo laminar a temperatura ambiente por un tiempo de tres 3 horas, posteriormente se almaceno en bolsas de papel, se marcó y se rotulo en bolsa plástica, para dejar en nevera a temperatura de $-25^{\circ} \mathrm{C}+/-5^{\circ} \mathrm{C}$.

\section{Elaboración de Extractos}

Se tomó el vestigio de la mancha preparada de $0,5 \mathrm{~cm} \times 0,5 \mathrm{~cm}$ dentro de un tubo debidamente marcado, se adicionaron $100 \mathrm{ul}$ de solución salina $0,85 \%$, a continuación, se tapó y se pasó a mezclar en el vortex por un tiempo de 30 s a velocidad de 1500rpm (revoluciones por minuto). El almacenamiento se llevó a cabo a temperatura de $5^{\circ} \mathrm{C}+/-3^{\circ} \mathrm{C}$ en nevera por 24 horas [16].

\section{J. Elaboración de Diluciones}

En la Tabla II se indican las diluciones de sangre humana en

TABLA II

DILUCIÓN DE SANGRE EN MANCHA

\begin{tabular}{|c|c|c|c|c|}
\hline Dilución & Sustrato & $\begin{array}{c}\text { Volumen de la } \\
\text { dilución (ul) }\end{array}$ & Más & $\begin{array}{c}\text { Solución salina } \\
\mathbf{0 . 8 5 \%} \text { (ul) }\end{array}$ \\
\hline $1: 10$ & $\begin{array}{l}\text { Papel } \\
\text { filtro }\end{array}$ & $\begin{array}{l}\text { 10ul extracto de } \\
\text { mancha }\end{array}$ & + & 90ul \\
\hline $1: 20$ & $\begin{array}{l}\text { Papel } \\
\text { filtro }\end{array}$ & $\begin{array}{c}\text { 50ul de dilución } \\
1: 10\end{array}$ & + & 50ul \\
\hline $1: 40$ & $\begin{array}{l}\text { Papel } \\
\text { filtro }\end{array}$ & $\begin{array}{c}\text { 50ul de dilución } \\
1: 20\end{array}$ & + & 50ul \\
\hline $1: 80$ & $\begin{array}{l}\text { Papel } \\
\text { filtro }\end{array}$ & $\begin{array}{c}\text { 50ul de dilución } \\
1: 40\end{array}$ & + & 50ul \\
\hline $1: 100$ & $\begin{array}{l}\text { Papel } \\
\text { filtro }\end{array}$ & $\begin{array}{l}\text { 10ul extracto de } \\
\text { mancha }\end{array}$ & + & 990ul \\
\hline
\end{tabular}

mancha, desde 1:10 hasta 1:100 procedente de un extracto de $0,5 \mathrm{~cm} \times 0,5 \mathrm{~cm}$ con $20 \mathrm{ul}$ de solución salina $0,85 \%$ y fueron realizadas de la siguiente manera. 
En la tabla III se presentan las diluciones en sangre liquida realizadas a partir de sangre total anti-coagulada con citrato de sodio.

\section{MetodologíA}

Para el estudio de las 100 muestras con clasificación cruzada se realiza un corrido por cada 10 muestras, teniendo como control positivo sangre humana y como control negativo ácido acético glacial, se toma una lámina portaobjeto y se adiciona 10ul de la muestra o extracto a estudiar, se pasa a secar al horno por un tiempo de 6 t/- 2 minutos a temperatura de $60^{\circ} \mathrm{C}$, seguidamente se coloca en cámara de extracción y se adiciona 10ul más de la muestra en estudio , se pasa a secar nuevamente la muestra al horno por segunda vez en un tiempo de $5+/-2$ minutos a $60^{\circ} \mathrm{C}$, se deja en cámara de extracción y se adiciona $20 u l$ de ácido acético glaciar puro, se realiza un pequeño contacto de la muestra con un cubreobjeto y se deja caer lentamente sobre ella,

\begin{tabular}{cccc}
\hline \hline Dilución & $\begin{array}{c}\text { Volumen de la dilución } \\
\text { (ul) }\end{array}$ & Más & $\begin{array}{c}\text { Solución salina } \\
\mathbf{0 . 8 5 \% ( u l )}\end{array}$ \\
\hline $1: 10$ & 10ul sangre & + & $90 \mathrm{ul}$ \\
$1: 20$ & 50ul de dilución 1:10 & + & $50 \mathrm{ul}$ \\
$1: 40$ & 50ul de dilución 1:20 & + & $50 \mathrm{ul}$ \\
$1: 80$ & 50ul de dilución 1:40 & + & $50 \mathrm{ul}$ \\
1:100 & 10ul sangre & + & $990 \mathrm{ul}$ \\
1:160 & 50ul de dilución 1:80 & + & $50 \mathrm{ul}$ \\
1:200 & 500ul de dilución 1:100 & + & $500 \mathrm{ul}$ \\
\hline \hline
\end{tabular}

obteniendo de esta manera su cubrimiento total, se utiliza un mechero de alcohol y automáticamente se toma con una pinza metálica el portaobjeto calentando con suavidad, hasta obtener la formación de burbujas de gas en el líquido situado debajo del cubreobjetos, indicando de esta manera que el ácido acético ha llegado al punto de ebullición, posteriormente se deja la muestra enfriar por un tiempo de 5 +/- 1 minutos y se adiciona por capilaridad 20ul de ácido acético glacial para ser observada en el microscopio con el objetivo de 10X y en seguida en 40X obteniendo las lecturas correspondientes.

\section{A. Lectura de Resultados}

Positivo: Formación de cristales de hemina, coloración chocolate oscuro y en forma de pequeñas placas rómbicas que varían de tamaño de 5 +/- 10 um (micrómetros).

Negativo: Ausencia de estos cristales.

El análisis cualitativo y sus atributos numéricos alcanzados en sensibilidad, especificidad, valor predictivo positivo, valor predictivo negativo, índice de concordancia e índice de kappa según la matriz de confusión fueron adquiridos siguiendo el protocolo de análisis de datos estadísticos [17], también se mantuvieron presentes las normas de bioseguridad y limpieza del material manipulado por el laboratorio de biología forense.

\section{RESUltados y DiscucióN}

Utilizando la matriz de confusión, en la tabla IV se observan los datos obtenidos en la prueba de Teichmann

TABLA IV

PRUEBA DE TEICHMANN

\begin{tabular}{cccc}
\hline \hline PRUEBA & RESULTADOS & $\begin{array}{c}\text { MUESTRAS } \\
\text { POSITIVAS }\end{array}$ & $\begin{array}{c}\text { MUESTRAS } \\
\text { NEGATIVAS }\end{array}$ \\
\hline \multirow{2}{*}{ Teichmann } & POSITIVO & 39 & 0 \\
& NEGATIVO & 2 & 59 \\
\hline \hline
\end{tabular}

En la Tabla V, se presentan los datos obtenidos en el método de Teichmann

TABLA V

RESULTADOS ESTADÍSTICOS DE TEICHMANN

\begin{tabular}{lc}
\hline \hline \multicolumn{1}{c}{ Variable } & Método de Teichmann \\
\hline Sensibilidad & $96,22 \%$ \\
Especificidad & $100 \%$ \\
Valor Predictivo positivo & $100 \%$ \\
Valor Predictivo Negativo & $96,87 \%$ \\
Límite de Detección & $1 / 160$ (Sangre líquida) \\
Índice de Concordancia & $1 / 40$ Sangre mancha) \\
Índice de Kappa & $100 \%$
\end{tabular}

La especificidad obtenida por esta técnica, nos demuestra la capacidad para detectar como positivas aquellas muestras que realmente poseen el evento (o analito) en estudio, como también el grado de selectividad del método, obteniéndose un valor significativo en el análisis forense para la determinación de sangre.

La sensibilidad obtenida en la técnica de teichmann representa la probabilidad de encontrar como positivo una muestra que contenga realmente la sustancia buscada con un valor asertivo de confiabilidad para el analista forense.

El grado de concordancia realizados por los mismos analistas en el método de teichmann, con los mismos equipos de medición, en el mismo laboratorio evidenciaron que la técnica fue repetible y reproducible.

En el estudio del método de Teichmann con el propósito de evaluar sustancias o fluidos que interfieran y lleguen a generar resultados falsos positivos se elaboraron manchas sobre telas de algodón en vegetales como (remolacha, repollo morado, rábano, berenjena, pimentón, uyuco, plátano verde, tomate chonto, zanahoria, flor de fique); frutas (ciruela, papaya, manzana roja, mora, zapote, uva roja); productos químicos 
(detergente, hipoclorito, lugol, isodine, Tiosulfato de sodio, óxido de hierro, benzal); otras sustancias como (salsa de tomate, marcador rojo, esmalte rojo y vino rojo) y fluidos biológicos humanos( leche materna, semen, heces fecales, saliva y orina) [3].Donde en la técnica de Teichmann sustancias como el detergente y el hipoclorito debido a la acción inhibidora arrojan resultados falso negativos.

El valor de kappa obtenido por los dos analistas fue de 1, lo que muestra una buena concordancia entre analistas y que no se debió al azar; como también los valores operacionales obtenidos fueron iguales para los dos analistas.

\section{CONCLUSIONES}

En la investigación de manchas de sangre, el método confirmativo cristalográfico de teichmann sirve como soporte a las pruebas colorimétricas presuntivas debido a su alta especificidad y la poca cantidad de muestra que se necesita para realizar su marcha analítica.

Existen sustancias como el hidróxido de sodio, el hipoclorito que puede inhibir la reacción a pesar de existir sangre en la muestra. $\mathrm{El} \mathrm{Ph}$ fuertemente alcalino dado por el hidróxido de sodio como también el $\mathrm{Ph}$ acido del hipoclorito desnaturalizan las proteínas presentes en la hemoglobina impidiendo de esta manera la formación de cristales de hemina.

El uso de los diferentes sustratos utilizados (tela garza, jean, algodón, franela, lycra, lino, seda, papel filtro y dacron, todos ellos en colores como rojo, azul, amarillo y blanco), en el método de teichmann no infieren ni inhiben la reacción de cristalización.

En los distintos sustratos utilizados como (algodón, franela, jeans, lino, nylon, papel filtro, tela garza, dacrón, seda), el método de teichmann no presento ningún tipo de interferencia ni tampoco inhibieron las reacciones cristalográficas en el momento del análisis.

Los datos alcanzados de sensibilidad y límite de detección nos demuestran que la técnica queda limitada para estudio con muestras de una dilución de 1:160.

Las técnicas se repitieron por dos analistas el mismo día y días diferentes, demostrando que la prueba es repetible y reproducible, además se estableció que ni la temperatura de $25^{\circ} \mathrm{C}+/-5^{\circ} \mathrm{C}$, ni la humedad del lugar de análisis $\left(65^{\circ} \mathrm{C}+/-\right.$ $\left.5^{\circ} \mathrm{C}\right)$, mostraron algún efecto en el desarrollo del análisis.

La técnica presenta fortalezas como lo son el tiempo requerido para montar la prueba y obtener resultados por cada 10 muestras es de $40+/-5$ minutos y además se puede trabajar directamente sobre la mancha de sangre.

\section{REFERENCIAS}

[1] A. N. Coterhuanco, "Valoración de dos métodos colorimétricos para la detección de sangre en manchas secas en diferentes soportes y condiciones ambientales con fines forenses (Doctoral dissertation)," Bolivia , 2009.

[2] G. Calabuig, Medicina Legal y Toxicologia, Barcelona: Masson, 2004. ISBN: 9788445814154.

[3] G. J. Villanueva, Medicina Legal y Toxicologia, Barcelona,España: Masson. ISBN: 9788445814154, 2004

[4] C. E. A. N.K, "Manual Hyland de Inmunohematologia revisión,concisa de principios y procedimientos,", Angeles ,California EUA: Asistencia editorial del Dr. Jhon W.Palmer.

[5] R. E. Gaensslen, Sourcebook in Forensic Serology, Immunology, and Biochemistry, Washington, DC : US Department of Justice National Institute of Justice, 1983

[6] R. E. Gaensslen, de Sourcebook in forensic serology, immunology, and biochemistry, Washington, DC, US Department of Justice, National Institute of Justice, 1983, p. 79.

[7] P. Castelló, Revisión crítica del diagnóstico de orientación en el estudio de las manchas de sangre: falsos negativos en la prueba de Adler.Una aportación de la Qjuímica Legal, Valencia,España : Universidad de Valencia,Facultad de Ciencias Quimicas, 1997.

[8] M. H, “"The Haemochromogen Cristal Test For Blood,", The Britise Medical Jornal,, vol. 1, nº 134-6, p. 3395, 1926. PMID: 20772327.

[9] C. R. Nancy, Validadcion Tecnica Cristalografica Para Identificar Sangre en Diferentes Muestras Mediante El Reactivo de Takayama en el LBF Regional Occidente de Pereira,, Pereira, 2007.

[10] M. M. V. Silvia, ““VValidacion del Metodo de Thevenon Roland Como Prueba Para Detectar la Presencia de Sangre en Manchas,"," Instituto de Medicina Legal de Medellin, Medellin, 2002.

[11] I. N. d. M. L. y. C. Forenses, “" “DRC-GBF-T-IPR-05,”,” Pereira, 2004.

[12] J. N. Rodriguéz, “““Aportes de la hematología al campo forense: pruebas de orientación y de certeza.,"," Revista Skopein, vol. 4, n 13, pp. 35-36, 2016. ISSN 2346-9307.

[13] H. B. M. H. M. L. C. Vela, "Identificacion Forense de Manchas de sangre por Abacard Hematrace.Teichman y Takayama," de Universidad Autonoma de Chiapas. Facultad de Ciencias Quimicas . Campus IV. XXII Jornadascientificas, mexico,ISSN1946-5351, 2014.

[14] J. N. Rodriguez, "Aporte de la Hematologia al campo forense: pruebas de orientacion y de certeza," Skopen, vol. IV, n 13 ,ISSN: 2346-9307, pp. 35-37, 2016.

[15] V. G. Aubrey, "The use of Takayama Solution in the Identification of Blood Stains," The British Medical Journal, vol. 1, no 3724, pp. 932933, Mayo 21,1932. DOI: 10.1136/bmj.1.

[16] I. N. d. M. Legal, "PET sobre la determinacion de la actividad de Peroxidasas,” Instituto Nacional de Medicina Legal, Bogota, 2004.

[17] H. Manrique, "Manual de Referencia para la Validacion de Tecnicas Analiticas.Instituto Nacional De Medicina Legal y Ciencias Forenses.," Medellin,Colombia, 1995. 


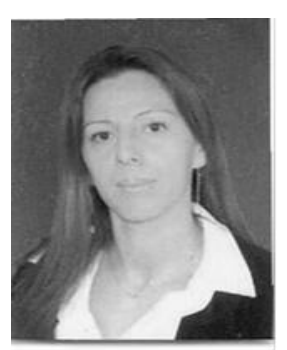

Nancy Janet Castillo Rodríguez nació en Cali, Colombia, en 1978. Recibió los títulos de Tecnóloga Química, Química Industrial Y Magister en Instrumentación Física por la universidad Tecnológica de Pereira (UTP), Pereira, en 1997,2008 y 2017. Actualmente Realiza sus estudios en Maestría en Enseñanza de la Física en la Universidad Tecnológica de Pereira, Desde 1998 se ha desempeñado como docente en el municipio de Pereira, Desde 2011 ha sido profesora en el Departamento de Física, UTP, y desde 2015 es profesora transitoria de medio tiempo. Sus intereses de investigación actuales incluyen proceso de tratamiento digital de imágenes en el campo de la medicina forense, identificación de fluidos biológicos en dictámenes de carácter forense y análisis de sustancias orgánicas. Pertenece al grupo de investigación Robótica Aplicada de la UTP.

ORCID: https://orcid.org/0000-0003-0856-0582

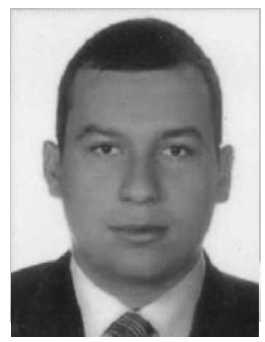

Sebastián Martínez García nació en Cartago, Colombia, en 1988. Recibió los títulos de Ingeniero Físico y Magister en Instrumentación Física por la Universidad Tecnológica de Pereira (UTP), Pereira, en 2011 y 2014. Actualmente realiza sus estudios en Doctorado en Ciencias en el área de Física en la Universidad Tecnológica de Pereira, Desde 2012 se ha desempeñado como docente en la Facultad de Ingenierías en el Programa de Ingeniería Física en la UTP, siendo actualmente profesor transitorio de medio tiempo. Sus intereses de investigación actuales incluyen desarrollo de prototipos para la experimentación, instrumentación, tratamiento digital de imágenes y aprendizaje automático. Pertenece al grupo de Investigación Diseño y Construcción de Prototipos para Experimentos de Demostración - DICOPED de la UTP. Paper Award in 2011.

ORCID: https://orcid.org/0000-0003-2055-5620 